Pacific Journal of Mathematics

A CONVOLUTION RELATED TO GOLOMB'S ROOT
FUNCTION FUN CHON 


\title{
A CONVOLUTION RELATED TO GOLOMB'S ROOT FUNCTION
}

\author{
E. E. GUERIN
}

The root function $\gamma(n)$ is defined by Golomb for $n>1$ as the number of distinct representations $n=a^{b}$ with positive integers $a$ and $b$. In this paper we define a convolution $\nabla$ such that $\gamma$ is the $\nabla$-analog of the (Dirichlet) divisor function $\tau$. The structure of the ring of arithmetic functions under addition and $\nabla$ is discussed. We compute and interpret $\nabla$ analogs of the Moebius function and Euler's $\Phi$-function. Formulas and an algorithm for computing the number of distinct representations of an integer $n \geqq 2$ in the form ${ }^{a}{ }_{k}$

$n=a_{1}^{a_{2}} \cdot{ }^{\cdot} \quad$, with $a_{i}$ a positive integer, $i=1, \cdots, k$, are given.

1. Introduction. Let $Z$ denote the set of positive integers, let $A$ denote the set of arithmetic functions (complex-valued functions with domain $Z$ ), and let $F$ denote the set of elements of $Z$ which are not $k$ th powers of any positive integer for $k>1(k \in Z)$. Note that $1 \notin F$. The divisor function $\tau$ can be defined as $\tau=\nu_{0} * \nu_{0}$, where $\nu_{0} \in A, \nu_{0}(n)=1$ for all $n \in Z$, and $*$ is the Dirichlet convolution defined for $\alpha, \beta \in A$ by $(\alpha * \beta)(n)=\sum_{d \mid n} \alpha(d) \beta(n / d)$.

Any integer $n \geqq 2$ having canonical form $n=p_{1}^{e_{1}} \cdots p_{r}^{e_{r}}$ is uniquely expressible as $n=m^{g}$, where $g=$ g.c.d. $\left(e_{1}, \cdots, e_{r}\right)$ and $m \in F$. Golomb [1] defines the root function $\gamma(n)$ for $n \in Z, n>1$, as the number of distinct representations $n=a^{b}$ with $a, b \in Z$; and he notes that $\gamma(n)=\tau(g)$ for $n=m^{g}, m \in F, g \in Z$. We let $\gamma(1)=1$.

For $\alpha, \beta \in A, n=m^{g}$, with $m \in F, g \in Z$, we define the $G$-convolution ("Golomb" convolution), $\nabla$, by

$$
(\alpha \nabla \beta)(n)=\sum_{d \mid g} \alpha\left(m^{d}\right) \beta\left(m^{g / d}\right) .
$$

We define $(\alpha \nabla \beta)(1)=1$. This $G$-convolution is not of the Narkiewicz type $[2,4]$.

In $\S 2$, we show that $\{A,+, \nabla\}($ where $(\alpha+\beta)(n)=\alpha(n)+\beta(n)$, $n \in Z)$ is a commutative ring with unity and we characterize the units and the divisors of zero. We define a $G$-multiplicative function and note that the set of $G$-multiplicative units in $\{A,+, \nabla\}$ forms an Abelian group under the operation $\nabla$.

We choose to define $\nabla$ as in (1.1) because then $\left(\nu_{0} \nabla \nu_{0}\right)(n)$ equals $\gamma(n)$, the number of distinct representations of $n$ as $a^{b}, a, b \in Z$; 
this is an analog of $\tau(n)=\left(\nu_{0} * \nu_{0}\right)(n)$ which is the number of distinct representations of $n$ as $a \cdot b, a, b \in Z$. In $\S 3, \nabla$-analogs of the Moebius function $\mu$, the sum of divisors function $\sigma$, and Euler's $\phi$-function are computed and interpreted.

In $\S 4$, we state formulas and an algorithm for computing the number of distinct representations of an integer $n \geqq 2$ in the form

$$
n=a_{1}^{a_{2} \cdot \cdot \cdot{ }^{a_{k}}}
$$

with $a_{i} \in Z, i=1, \cdots, k$.

2. The ring $\{A,+, \nabla\}$. First we state some properties related to the $G$-convolution.

THEOREM 2.1. (i ) The system $\{A,+, \nabla\}$ is a commutative ring with unity $\varepsilon_{\nabla}$ (where $\varepsilon_{\nabla}(n)=1$ if $n=1$ or $n \in F, \varepsilon_{\nabla}(n)=0$ otherwise).

(ii) $\alpha$ is a unit in $\{A,+, \nabla\}$ if and only if $\alpha(1) \neq 0$ and $\alpha(m) \neq 0$ for all $m \in F$.

(iii) $A$ nonzero arithmetic function $\alpha$ is a nonzerodivisor in $\{A,+, \nabla\}$ if and only if $\alpha(1) \neq 0$ and for each $m \in F$ there is a positive integer $g$ such that $\alpha\left(m^{g}\right) \neq 0$.

Proof. (i ) The associativity of $\nabla$ follows from (1.1) and the associativity of the Dirichlet convolution $*$. The commutativity of $\nabla$ and the distributivity of $\nabla$ over + follow directly from the definition of the $G$-convolution. If $n=m^{g}, g \in Z, m \in F$, then $\left(\varepsilon_{\nabla} \nabla \alpha\right)(n)=$ $\sum_{d \mid g} \varepsilon_{\nabla}\left(m^{d}\right) \alpha\left(m^{g / d}\right)=\alpha\left(m^{g}\right)=\alpha(n) ; \quad\left(\varepsilon_{\nabla} \nabla \alpha\right)(1)=\alpha(1)$. Therefore, $\varepsilon_{\nabla}$ is the unity element in $\{A,+, \nabla\}$.

(ii) An element $\beta$ in $A$ such that $\alpha \nabla \beta=\varepsilon_{\nabla}$ is defined if and only if $\alpha(1) \beta(1)=1, \alpha(m) \beta(m)=1$ for $m \in F$, and $\sum_{d \mid g} \alpha\left(m^{d}\right) \beta\left(m^{g / d}\right)=0$ for $m \in F, g \in Z, g>1$. Thus, $\alpha(1) \neq 0, \alpha(m) \neq 0$ for $m \in F$, if and only if $\alpha$ is a unit in $\{A,+, \nabla\}$.

(iii) If $\alpha(1)=0$, define $\beta \in A$ by $\beta(1)=1, \beta(n)=0$ if $n>1$. Then $(\alpha \nabla \beta)(n)=0$ for every $n \in Z$ and $\alpha$ is a divisor of zero. If there exists an $m \in F$ such that $\alpha\left(m^{g}\right)=0$ for every $g \in Z$, define $\beta \in A$ by $\beta(m)=1, \beta(n)=0$ for $n \in Z, n \neq m$. Then $(\alpha \nabla \beta)(n)=0$ for all $n \in Z$ and $\alpha$ is a divisor of zero.

Assume that $\alpha$ is a zero divisor in $\{A,+, \nabla\}$. Then there is some $\beta \in A, \beta \neq \bar{O}$ (where $\bar{O}(n)=0$ for all $n \in Z$ ), such that $\alpha \nabla \beta=\bar{O}$. (1) If $\beta(1) \neq 0$ then $\alpha \nabla \beta=\bar{O}$ implies that $\alpha(1) \beta(1)=0$ and that $\alpha(1)=0$. (2) If $\beta(1)=0$, let $n$ be the smallest positive integer such that $\beta(n) \neq 0$; if $n=m^{v}, m \in F, v \in Z$, we show that $\alpha\left(m^{w}\right)=0$ for all $w \in Z$. First, $(\alpha \nabla \beta)\left(m^{v}\right)=\sum_{d \mid v} \alpha\left(m^{d}\right) \beta\left(m^{v / d}\right)=0$ implies that 
$\alpha(m) \beta\left(m^{v}\right)=0$ and that $\alpha(m)=0$. And $(\alpha \nabla \beta)\left(m^{2 v}\right)=0$ implies that $\alpha(m) \beta\left(m^{2 v}\right)+\alpha\left(m^{2}\right) \beta\left(m^{v}\right)=0$ and so $\alpha\left(m^{2}\right)=0$. Assume that $\alpha\left(m^{t}\right)=$ $0,1 \leqq t<r$. Then $(\alpha . \nabla \beta)\left(m^{r v}\right)=\sum_{d \mid r v} \alpha\left(m^{d}\right) \beta\left(m^{r v / d}\right)=0$ implies that $\alpha\left(m^{r}\right) \beta\left(m^{v}\right)=0$ and $\alpha\left(m^{r}\right)=0$. Therefore, $\alpha\left(m^{w}\right)=0$ for all $w \in Z$ by induction. This completes the proof of the theorem.

We define $\alpha \in A$ to be $G$-multiplicative if $\alpha(1)=1$, and whenever $(a, b)=1$ and $m \in F, \alpha \cdot\left(m^{a b}\right)=\alpha \cdot\left(m^{a}\right) \alpha\left(m^{b}\right)$.

THEOREM 2.2. The set of G-multiplicative functions which are units in $\{A,+, \nabla\}$ form an abelian group under $\nabla$.

Proof. If $\alpha$ and $\beta$ are $G$-multiplicative, then $\alpha \nabla \beta$ is also; the proof is similar to that of the multiplicativity of $\alpha * \beta$ given that $\alpha$ and $\beta$ are multiplicative [3, p. 93]. It is then easy to verify the required group properties.

3. The functions $\sigma_{\nabla}, \mu_{\nabla}, \phi_{\nabla}$. As noted earlier, $\gamma=\nu_{0} \nabla \nu_{0}$ is the $\nabla$-analog of $\tau=\nu_{0} * \nu_{0}$. For example, $\gamma(64)=\gamma\left(2^{6}\right)=\tau(6)=4$, and 64 can be represented in the form $a^{b}$ for $a, b \in Z$ in four ways: $\left(2^{1}\right)^{6}=$ $2^{6},\left(2^{2}\right)^{3}=4^{3},\left(2^{3}\right)^{2}=8^{2}$, and $\left(2^{6}\right)^{1}=64^{1}$.

If we define $\sigma_{\bar{\nabla}}$ by $\sigma_{\bar{\nabla}}=\nu_{0} \nabla \nu_{1}$, then for $n=m^{g}, m \in F, g \in Z$, $\sigma_{i}(n)=\sum_{a \mid g} m^{d}$. So $\sigma_{\bar{\nu}}(n)$ is the sum of the $a$ 's such that $a^{b}=n$, whereas $\sigma(n)=\left(\nu_{0} * \nu_{1}\right)(n)$ is the sum of the $a$ 's such that $a \cdot b=$ $n(a, b \in Z)$.

An analog $\mu_{\nu}$ of the Moebius function $\mu$ (where $\mu$ satisfies $\nu_{0} * \mu=\varepsilon$ with $\varepsilon(1)=1, \varepsilon(n)=0$ otherwise) is defined by $\nu_{0} \nabla \mu_{\nabla}=\varepsilon_{\nabla}$. Then $\mu_{\nabla}(n)=1$ if $n=1, \mu_{\nabla}(n)=\mu(g)$ if $n=m^{g}, m \in F, g \in Z$.

Euler's $\phi$-function, which satisfies $\phi=\mu * \nu_{1}$ (where $\nu_{1}(n)=n$ for all $n \in Z)$, has an analog $\phi_{\nabla}$ with $\phi_{\nabla}(1)=1, \phi_{\nabla}(n)=\left(\mu_{\nabla} \nabla \nu_{1}\right)(n)=$ $\sum_{d \mid g} \mu(d) m^{g / d}$ for $n=m^{g}, m \in F, g \in Z$. Thus, $\phi_{V}(m)=m$ for $m \notin F$ and $\dot{\phi}_{i}\left(m^{p}\right)=m^{p}-m$ for $m \in F, p$ prime. If $n=m^{g}, m \in F, g \in Z$, then $\phi_{i}(n)$ is $n$ minus the number of positive integers less than or equal to $n$ which are expressible as $r^{d}, r \in Z, d \mid g, d>1$. Here, $n$ and $r^{d}$ have a common power $d>1$ (since $n=a^{d}$ with $a=m^{g / d}$ ); this corresponds, in the computation of $\phi(n)$, to nonrelativity-prime $n$ and $m$ having a common divisor $d>1$. To illustrate, $\phi_{V}(64)=$ $2^{6}-2^{3}-2^{2}+2^{1}=64-10=54$. The ten integers of the form $r^{d}$, $r \in Z, d \mid 6, d>1, r^{d} \leqq 64$, are

$$
1^{2}, 2^{2}, 3^{2}, 4^{2}, 5^{2}, 6^{2}, 7^{2}, 8^{2}=4^{3}=2^{6}, 2^{3}, 3^{3} \text {. }
$$

And, for example, $3^{2}$ and $n=8^{2}$ have common power 2 , while $2^{3}$ and $n=4^{3}$ have common power 3 .

It can be verified that $\gamma, \varepsilon_{\nabla}, \nu_{0}$, and $\mu_{\nabla}$ are $G$-multiplicative functions whereas $\nu_{1}, \sigma_{\nabla}$, and $\phi_{\nabla}$ are not. 
If $n=m^{g}, m \in F, g \in Z$, then $\sigma_{\nabla}(n)=2 n$ has no solutions. But if we define a $G$-perfect number $n=m^{g}, m \in F, g \in Z$, as one such that $\Pi_{d \mid g} m^{d}=n^{2}$, then $n$ is $G$-perfect if and only if $g$ is perfect if and only if $\left(\nu_{0} * \nu_{1}\right)(g)=2 g$.

4. Power representations of $n$. If $n=m^{g}, m \in F, g \in Z$, define $\rho \in A$ by $\rho(n)=g$; define $\rho(1)=1$. Then $\gamma(n)=\tau(\rho(n))=\left(\nu_{0} \nabla \nu_{0}\right)(n)=$ $\left(\left(\nu_{0} * \nu_{0}\right) \circ \rho\right)(n) \quad($ where $(\alpha \circ \beta)(n)=\alpha(\beta(n)))$. We note that $\mu_{\nabla}(n)=\mu(\rho(n))$ and $\varepsilon,(n)=\varepsilon(\rho(n))$.

Let $R_{k}(n)$ denote the number of distinct representations of $n=$ $m^{g}, m \in F, g \in Z$, in the form given in (1.2). (Assume that $R_{k}(1)=1$ for all $k \in Z$.) We have the following formulas.

$$
\begin{aligned}
R_{1}(n) & =1 . \\
R_{2}(n) & =\gamma(n)=\tau(\rho(n))=\left(\nu_{0} \nabla \nu_{0}\right)(n) . \\
R_{3}(n) & =\sum_{d \mid g} \gamma(d)=\sum_{d \mid \rho(n)} \tau(\rho(d))=\left(\nu_{0} *(\tau \circ \rho)\right)(\rho(n)) \\
& =\left(\left(\nu_{0} *\left(\nu_{0} \nabla \nu_{0}\right)\right) \circ \rho\right)(n) . \\
R_{4}(n) & =\sum_{d \mid g} \sum_{r \mid \rho(d)} \gamma(r)=\sum_{d \mid \rho(n)} \sum_{r \mid \rho(d)} \tau(\rho(r))=\left(\nu_{0} *\left(\left(\nu_{0} *(\tau \circ \rho)\right) \circ \rho\right)\right)(\rho(n)) \\
& =\left(\left(\nu_{0} *\left(\left(\nu_{0} *\left(\nu_{0} \nabla \nu_{0}\right)\right) \circ \rho\right)\right) \circ \rho\right)(n) .
\end{aligned}
$$

Similar formulas can be written for $R_{k}(n)$ for any $k \in Z$.

If $n>1$, then $R_{k}(n)$ can be computed as follows. List $d_{1}$ such that $d_{1} \mid g$, list $\rho\left(d_{1}\right)$, list $d_{2}$ such that $d_{2} \mid \rho\left(d_{1}\right)$, list $\rho\left(d_{2}\right), \cdots$, list $d_{k-2}$ such that $d_{k-2} \mid \rho\left(d_{k-3}\right)$, list $\rho\left(d_{k-2}\right)$; and $R_{k}(n)$ is the sum of the number of divisors of the entries in the final list.

For example, if $n=20^{400}, g=\rho(n)=2^{4} \cdot 5^{2}$. For $d_{1}\left|g, d_{2}\right| \rho\left(d_{1}\right)$, $d_{3} \mid \rho\left(d_{2}\right)$, we have these lists.

$$
\begin{aligned}
d_{1} & =1,2,4,8,16,1 \cdot 5,2 \cdot 5,4 \cdot 5,8 \cdot 5,16 \cdot 5,1 \cdot 5^{5}, 2 \cdot 5^{2}, 4 \cdot 5^{2}, 8 \cdot 5^{2}, 16 \cdot 5^{2} \\
\rho\left(d_{1}\right) & =1,1,2,3,4, \quad 1,1,1,1,1,2, \quad 1, \quad 2, \quad 1,2 \\
d_{2} & =1,1,1,2,1,3,1,2,4, \quad 1,1,1,1,1,1,2,1,1,2,1,1,2 \\
\rho\left(d_{2}\right) & =1,1,1,1,1,1,1,1,2, \quad 1,1,1,1,1,1,1,1,1,1,1,1,1 \\
d_{3} & =1,1,1,1,1,1,1,1,1,2,1,1,1,1,1,1,1,1,1,1,1,1,1 \\
\rho\left(d_{3}\right) & =1,1,1,1,1,1,1,1,1,1,1,1,1,1,1,1,1,1,1,1,1,1,1
\end{aligned}
$$

Then $R_{3}\left(20^{400}\right)=2 \tau(1)+\tau(2)+\tau(3)+\tau(4)+5 \tau(1)+\tau(2)+\tau(1)+\tau(2)+$ $\tau(1)+\tau(2)=22$. And $R_{4}\left(20^{400}\right)=23, R_{5}\left(20^{400}\right)=23$; in fact, $R_{k}\left(20^{400}\right)=$ 23 for $k \geqq 4$. There are four representations of $n=20^{400}$ in the form given in (1.2) for $k=4$ which correspond to $d_{1}=16$ (since $\tau(1)+\tau(1)+\tau(2)=4)$. They are 


$$
a^{16^{1}}, \quad a^{2^{1}}, \quad a^{2^{4^{1}}}, a^{2^{2}},
$$

where $a=335,544,320,000,000,000,000,000,000,000,000$ (which is $20^{25}$ in expanded form). In only one of these representations is $a_{i} \neq 1, i=1, \cdots, 4$. In general, the number of distinct representations of $n=m^{g}, m \in F, g \in Z$, in the form given in (1.2) with the additional requirement that $a_{i} \neq 1, i=1, \cdots, k$, is the sum of the number of divisors less one of the entries in the final list (for $\left.\rho\left(d_{k-2}\right)\right)$.

\section{REFERENCES}

1. S. W. Golomb, A new arithmetic function of combinatorial significance, J. Number Theory, 5 (1973), 218-223.

2. W. Narkiewicz, On a class of arithmetical convolutions, Colloq. Math., 10 (1963), 81-94.

3. I. Niven and H. Zuckerman, An Introduction to the Theory of Numbers, 3rd ed., New York, John Wiley Sons, 1972.

4. M. V. Subbarao, On Some Arithmetic Convolutions in The Theory of Arithmetic Functions, Lecture Notes \#251, New York, Springer-Verlag, 1972.

Received October 3, 1977

SETON HALL UNIVERSITY

SOUTh ORANGe, NJ 07079 



\section{PACIFIC JOURNAL OF MATHEMATICS}

\section{EDITORS}

RichaRd ARENS (Managing Editor)

University of California

Los Angeles, CA 90024

Charles W. Curtis

University of Oregon

Eugene, OR 97403

C. C. Moore

University of California

Berkeley, CA 94720

\section{J. DUGUNDJI}

Department of Mathematics

University of Southern California

Los Angeles, CA 90007

R. FinN and J. Milgram

Stanford University

Stanford, CA 94305

\section{E. F. BECKENBACH \\ B. H. NeumanN

\author{
UNIVERSITY OF BRITISH COLUMBIA \\ CALIFORNIA INSTITUTE OF TECHNOLOGY \\ UNIVERSITY OF CALIFORNIA \\ MONTANA STATE UNIVERSITY \\ UNIVERSITY OF NEVADA, RENO \\ NEW MEXICO STATE UNIVERSITY \\ OREGON STATE UNIVERSITY \\ UNIVERSITY OF OREGON
}

F. WOLF

K. Yoshida

\section{SUPPORTING INSTITUTIONS}

\author{
UNIVERSITY OF SOUTHERN CALIFORNIA \\ STANFORD UNIVERSITY \\ UNIVERSITY OF HAWAII \\ UNIVERSITY OF TOKYO \\ UNIVERSITY OF UTAH \\ WASHINGTON STATE UNIVERSITY \\ UNIVERSITY OF WASHINGTON
}

The Supporting Institutions listed above contribute to the cost of publication of this Journal, but they are not owners or publishers and have no responsibility for its content or policies.

Mathematical papers intended for publication in the Pacific Journal of Mathematics should be in typed form or offset-reproduced, (not dittoed), double spaced with large margins. Please do not use built up fractions in the text of the manuscript. However, you may use them in the displayed equations. Underline Greek letters in red, German in green, and script in blue. The first paragraph or two must be capable of being used separately as a synopsis of the entire paper. Items of the bibliography should not be cited there unless absolutely necessary, in which case they must be identified by author and journal, rather than by item number. Manuscripts, in triplicate, may be sent to any one of the editors. Please classify according to the scheme of Math. Reviews, Index to Vol. 39. All other communications should be addressed to the managing editor, or Elaine Barth, University of California, Los Angeles, California, 90024.

50 reprints to each author are provided free for each article, only if page charges have been substantially paid. Additional copies may be obtained at cost in multiples of 50 .

The Pacific Journal of Mathematics is issued monthly as of January 1966. Regular subscription rate: $\$ 72.00$ a year (6 Vols., 12 issues). Special rate: $\$ 36.00$ a year to individual members of supporting institutions.

Subscriptions, orders for numbers issued in the last three calendar years, and changes of address should be sent to Pacific Journal of Mathematics, P.O. Box 969, Carmel Valley, CA 93924, U.S.A. Older back numbers obtainable from Kraus Periodicals Co., Route 100, Millwood, NY 10546.

PUBLISHED BY PACIFIC JOURNAL OF MATHEMATICS, A NON-PROFIT CORPORATION

Printed at Kokusai Bunken Insatsusha (International Academic Printing Co., Ltd.). 8-8, 3-chome, Takadanobaba, Shinjuku-ku, Tokyo 160, Japan. 


\section{Pacific Journal of Mathematics \\ Vol. 79, No. $2 \quad$ June, 1978}

David R. Adams, Quasi-additivity and sets of finite $L^{p}$-capacity ........ 283

George M. Bergman and Warren Dicks, Universal derivations and universal

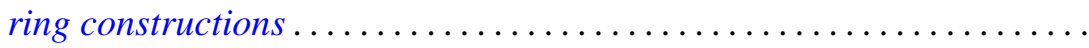

Robert F. Brown, Addendum to: "Fixed points of automorphisms of compact Lie groups".........................................

Eugene Frank Cornelius, Jr., Characterization of a class of torsion free

groups in terms of endomorphisms .......................

Andres del Junco, A simple measure-preserving transformation with trivial centralizer..................................... 357

Allan Lee Edmonds, Extending a branched covering over a handle ...... 363

Sjur Flam, A characterizaton of $\mathbf{R}^{2}$ by the concept of mild convexity .......

Claus Gerhardt, $L^{p}$-estimates for solutions to the instationary Navier-Stokes

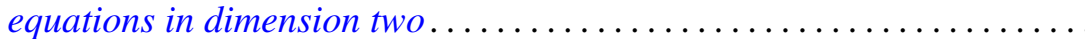

Kensaku Gomi, Finite groups with a standard subgroup isomorphic to

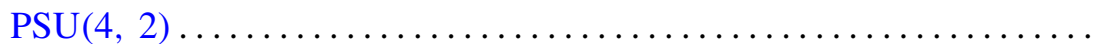

E. E. Guerin, A convolution related to Golomb's root function ........... 463

$\mathrm{H}$. B. Hamilton, Modularity of the congruence lattice of a commutative cancellative semigroup .................................

Stephen J. Haris, Complete reducibility of admissible representations over function fields.

Shigeru Itoh and Wataru Takahashi, The common fixed point theory of singlevalued mappings and multivalued mappings... ...

James E. Joseph, Multifunctions and graphs .............

Bruce Magurn, Images of $S K_{1} Z G$. .

Arnold Koster Pizer, A note on a conjecture of Hecke... .

Marlon C. Rayburn, Maps and h-normal spaces ...........

Barada K. Ray and Billy E. Rhoades, Corrections to: "Fixed-point theorems for mappings with a contractive iterate" ..............

Charles Irvin Vinsonhaler, Corrections to: "Torsion free abelian groups quasiprojective over their endomorphism rings. II". . . 